\title{
NEW TYPE OF NEARLY MONOTONIC PASSBAND FILTERS WITH SHARP CUTOFF
}

UDC (621.372.54:004.382):512.62

\author{
Dragana Živaljević, Saša V. Nikolić \\ University of Niš, Faculty of Electronic Engineering, Niš, Republic of Serbia
}

\begin{abstract}
New type of nearly monotonic rational function with one pair of zeros on imaginary axis has been proposed. By using these new functions with zeros as a parameter, it is possible to make tradeoff between minimum stopband attenuation and selectivity of the amplitude characteristic. In order to present efficiency of the proposed filter, comparison with allpole filter monotonic in the passband and inverse Chebyshev filter is presented. Also, design example for the seventh order new type lowpass filter is given.
\end{abstract}

Keywords: Legendre polynomial, Christoffel-Darboux identity, orthogonal functions, allpole filters, LC ladder network, lossless networks.

\section{INTRODUCTION}

Application of classical orthogonal polynomials in designing the analog lowpass filters is described in many practical solutions [1,2,3]. All-pole lowpass filters, which transfer functions have all zeros at the infinity, are easier for implementation than the lowpass transfer functions with finite zeros on the imaginary axis, as for example inverse Chebyshev or Elliptic filters [1]. Therefore, all-pole approximations are always considered as a first option in filter design.

Many approximations offer excellent magnitude characteristic at the expense of the group delay characteristic, as Chebyshev [1] and Legendre [4, 5] filters. From the other side, there are approximations optimized for maximally-flat constant group delay characteristic as Bessel filter [6]. As a compromise, filters with monotonic passband magnitude characteristic achieve exchange between magnitude and phase characteristics. The Legendre-Papuolis (known as an "Optimum L" or just "Optimum") filter proposed by A. Papuolis [5] with critical monotonic magnitude response in the passband, has the maximum rolloff rate for a given filter degree. It provides a compromise between the Butterworth filter which is

Received February 24, 2017

Corresponding author: Dragana Živaljević

University of Niš, Faculty of Electronic Engineering, Aleksandra Medvedeva 14, 18000 Niš, Republic of Serbia

E-mail: dragana.zivaljevic@elfak.ni.ac.rs 
maximally flat and Legendre [4] with ripples in the passband. Legendre-Papoulis filters can be useful in applications that need a steep cutoff at the passband edge but cannot tolerate passband ripples, or in cases when Legendre filter produces high values of the group delay at the passband edge.

Recently, low pass filters having nearly monotonic pass-band magnitude response, designed by using the sum-of-square Legendre polynomials, have been proposed [7]. These filters have smaller pass-band magnitude distortion comparing to Legendre-Papoulis filters, but both filters have similar performance in the stop-band.

This paper proposes a design technique for the low-pass filters based on sum-of-squares Legendre polynomials with one pair multiple zeros on imaginary axis in order to provide steeper slopes at the cutoff frequency, but without influence to the group delay response. These filters are nearly monotonic or monotonic in the pass-band and non-monotonic in the stopband. The proposed design is compared with inverse Chebyshev filters which are maximally flat in the passband and equiripple in the stopband.

\section{APPROXIMATION}

The magnitude response of any allpole lowpass filter can be written in the form:

$$
|H(x)|=\frac{1}{\sqrt{1+\varepsilon^{2} \Psi\left(x^{2}\right)}},
$$

where $x$ is a frequency variable, $\varepsilon$ is constant which controls the maximum passband attenuation $a_{\max }$ (in $\mathrm{dB}$ ) as $\varepsilon=\sqrt{10^{0.1 a_{\max }}-1}$. The characteristic function $\Psi_{n}\left(x^{2}\right)$ is rational function normalized so that $\Psi_{n}(1)=1$.

When the voltage or current source is not ideal and the load is finite and nonzero, we have the situation of a doubly terminated lossless ladder network. The general form of the doubly resistively terminated two-port LC filter is shown in Fig. 1.

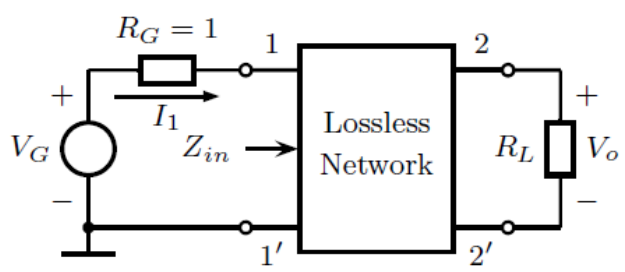

Fig. 1 Doubly terminated lossless network driven by voltage source.

In a doubly terminated network, the performance is measured by the ratio of power delivered to the load, $P_{L}$, and maximum power that can be delivered by the source, $P_{\max }$. This ratio defines the transmission coefficient $H(\mathrm{j} \omega)$ as

$$
\frac{P_{L}}{P_{\max }}=|H(\mathrm{j} \omega)|^{2}=1-|\Gamma(\mathrm{j} \omega)|^{2},
$$


where $\Gamma(j \omega)$ is the reflection coefficient looking toward the input of the filter network. It can be calculated as

$$
|\Gamma(\mathrm{j} \omega)|^{2}=\left.\Gamma(s) \Gamma(-s)\right|_{s=\mathrm{j} \omega}=\frac{\left|R_{G}-Z_{\text {in }}(\mathrm{j} \omega)\right|^{2}}{\left|R_{G}+Z_{\text {in }}(\mathrm{j} \omega)\right|^{2}},
$$

where the reference impedance is the source resistance, $R_{G}$, and $Z_{\text {in }}(j \omega)$ is the input impedance looking toward the lossless network (Fig.1) at port 1.

The characteristic function of the $\mathrm{n}$-th degree Legendre low-pass filter with single pair of zeros at $\pm j \omega_{0}$, where $\omega_{0}>1$, is:

$$
\psi_{n}\left(\omega^{2}\right)=\frac{L_{2 n}\left(\omega^{2}\right)}{L_{2 n}(1)}\left(\frac{\omega_{0}^{2}-1}{\omega^{2}-\omega_{0}^{2}}\right)^{2 m},
$$

where $L_{2 n}(\omega)$ is orthogonal polynomial Kernel obtained as

$$
L_{2 n}(\omega)=\bar{p}_{0}^{2}(\omega)+\bar{p}_{1}^{2}(\omega)+\ldots+\bar{p}_{n}^{2}(\omega),
$$

and $\bar{p}_{i}(\omega), i=1, \ldots, n$ are orthonormal Legendre polynomials of the first kind (entire even or odd) of $n$-th degree

$$
\bar{p}_{n}(\omega)=\sqrt{\frac{2 n+1}{2}} P_{n}(\omega),
$$

and $P_{n}(\omega)$ is the classical orthogonal Legendre polynomial. Since $P_{n}(1)=1$ then $L_{2 n}(1)=(n+1)^{2} / 2$.

By using Christoffel-Darboux formula [8] equation (5) is reduced to:

$$
L_{2 n}(\omega)=\frac{k_{n}}{k_{n+1}}\left[\frac{\mathrm{d} \bar{p}_{n+1}(\omega)}{\mathrm{d} \omega} \bar{p}_{n}(\omega)-\frac{\mathrm{d} \bar{p}_{n}(\omega)}{\mathrm{d} \omega} \bar{p}_{n+1}(\omega)\right],
$$

where $k_{n}$ is leading coefficient of $\bar{p}_{n}(\omega)$.

Performing analytic continuation on (1) resulting in

$$
H(s) H(-s)=\frac{\left(s^{2}+\omega_{0}^{2}\right)^{2 m}}{\frac{2 \varepsilon^{2}\left(\omega_{0}^{2}-1\right)^{2 m}}{(n+1)^{2}} L_{2 n}\left(s^{2}\right)+\left(s^{2}+\omega_{0}^{2}\right)^{2 m}} .
$$

From (8) $\mathrm{H}(\mathrm{s})$ can be writen as:

$$
H(s)=\frac{s^{2}+\omega_{0}^{2}}{D(s)}
$$

where $D(s)$ represents the left half-plane roots of

$$
\frac{2 \varepsilon^{2}\left(\omega_{0}^{2}-1\right)^{2 m}}{(n+1)^{2}} L_{2 n}\left(s^{2}\right)+\left(s^{2}+\omega_{0}^{2}\right)^{2 m}=0 .
$$

The attenuation versus normalized frequency $\omega$ of the seventh degree all-pole $(m=0)$ sum-of-squares Legendre polynomials $L_{n}(s)$ and two new functions of the same degree 
with $m=1\left(\omega_{0}=1.41442\right)$ and $m=2\left(\omega_{0}=2.0624\right)$, which provide minimum stopband attenuation $a_{\min }=50 \mathrm{~dB}$, are illustrated in Figure 2. As it is shown, a pair of zeros on the imaginary axis, which are placed in stop-band, decrease pass-band attenuation and increase stop-band attenuation, but they have not effect on the group delay.

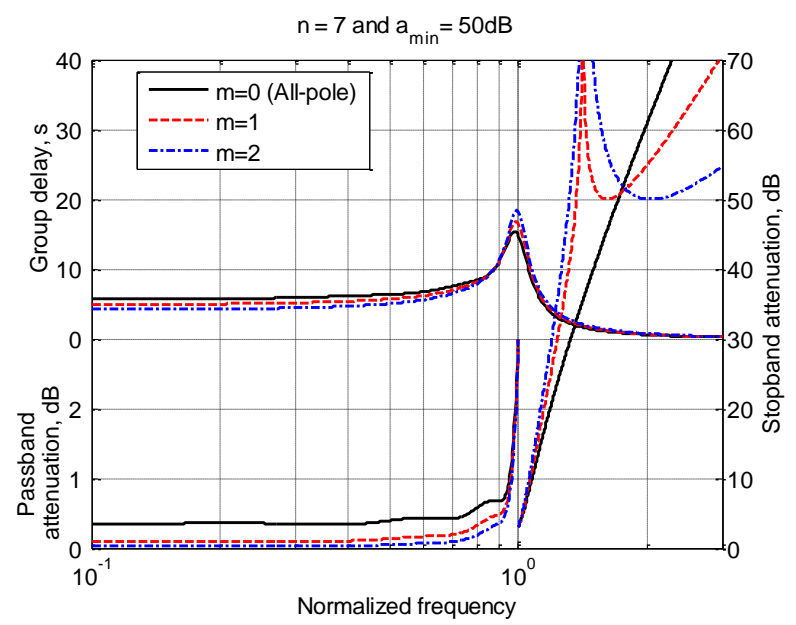

Fig. 2 Attenuation responses and group delay characteristic of the proposed Legendre filters for $n=7$.

A comparison of the steepness of cutoff of filters considered here, can be made by calculating the cutoff slopes,

$$
S=\frac{\mathrm{d}}{\mathrm{d} \omega}\left|H\left(\omega^{2}\right)\right|_{\omega=1}=-\left.\frac{\varepsilon^{2}}{2\left(\varepsilon^{2}+1\right)^{3 / 2}} \frac{\mathrm{d}}{\mathrm{d} \omega} \psi\left(\omega^{2}\right)\right|_{\omega=1}
$$

at the cutoff frequency $\omega_{\mathrm{c}}=1$ for equal attenuation in the passband $a_{\max }$ [9]. $\psi\left(\omega^{2}\right)$ is the characteristic function given by (1).

$$
\psi\left(\omega^{2}\right)=\frac{2 L_{2 n}\left(\omega^{2}\right)}{(n+1)^{2}}\left(\frac{\omega_{0}^{2}-1}{\omega^{2}-\omega_{0}^{2}}\right)^{2 m}=\left(\frac{\omega_{0}^{2}-1}{\omega^{2}-\omega_{0}^{2}}\right)^{2 m} \frac{2}{(n+1)^{2}} \sum_{i=0}^{n} \frac{2 i+1}{2} P_{i}^{2}(\omega) .
$$

By differentiating equation (12) with respect to $\omega$ it is shown that the cutoff slope at the frequency $\omega=1$ is given by

$$
\frac{\mathrm{d}}{\mathrm{d} \omega} \psi\left(\omega^{2}\right)=\frac{4 m}{\omega_{0}^{2}-\omega^{2}}\left(\frac{\omega_{0}^{2}-1}{\omega^{2}-\omega_{0}^{2}}\right)^{2 m}+\frac{2}{(n+1)^{2}} \sum_{i=0}^{n} \frac{2 i+1}{2} 2 P_{i}(\omega) \frac{\mathrm{d}}{\mathrm{d} \omega} P_{i}(\omega) .
$$

Since at $\omega=1$ are $P_{i}(1)=1$ and $\mathrm{d} P_{i}(\omega) /\left.\mathrm{d} \omega\right|_{\omega=1}=i(i+1) / 2$, then

$$
\frac{\mathrm{d}}{\mathrm{d} \omega} \psi\left(\omega^{2}\right)=\frac{4 m}{\omega_{0}^{2}-1}+\frac{2}{(n+1)^{2}} \sum_{i=0}^{n} \frac{(2 i+1) i(i+1)}{2}=\frac{n(n+2)}{2}+\frac{4 m}{\omega_{0}^{2}-1} .
$$


Equation (14) contains two parts. The first part is the cutoff slope of polynomial sumof-square Legendre filter. The second part shows how the cutoff slope of the final transfer function increases with finite transmission zero. The cutoff slope increases if the multiplicity of transmission zero, $m$, increases, and transmission zero, $\omega_{0}$, moves toward the passband edge. Note, that the stopband attenuation decreases when the transmission zero moves toward the passband edge.

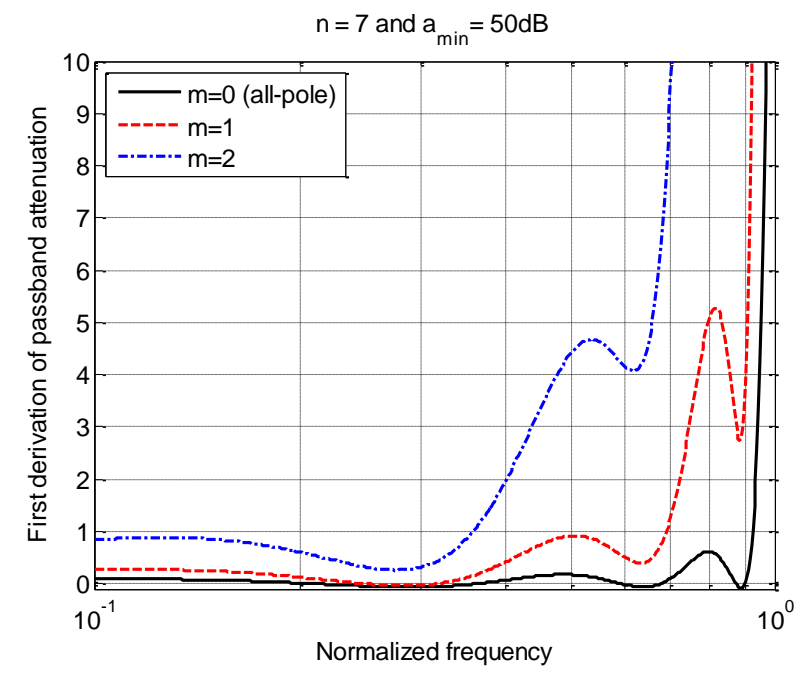

Fig. 3 First derivatives of the passband attenuation of the proposed filter for $n=7$ and $m=0, m=1$ and $m=2$.

In Figure 3 are shown the first derivatives of the passband attenuations of all transfer functions given in Figure 2. Negative derivatives (corresponding to decreasing parts of the magnitude characteristic) have very small values, smaller than 0.1 . If the stop band attenuation decreases and the multiplicity of zero increases, then the passband magnitude can be critical monotonic or monotonic. Also, it can be noted that these values are stable for all practical filter degrees.

\section{REALIZATION}

Doubly terminated passive LC ladder filters have transfer functions with low sensitivities to component variations in the passband [10]. The all-pole low-pass prototypes synthesis, given a prescribed insertion-loss between a resistive source and a resistive load, is a classical procedure presented in many textbooks on network synthesis [11-13]. Modifications of these prototypes enable the design of other filter types. On the other side, some methods transform an LC ladder network directly into a digital filter [14]. In this method an LC ladder network is transformed directly into a digital filter by first representing the ladder filter branch equations in flow graph form and then using the bilinear $z$-transform to transform the ladder elements into digital filter elements. 
The LC ladder network design technique is to make source impedance to unity, $R_{G}=1$, and pass-band edge to unity, $\omega_{\mathrm{c}}=1$, to transform specifications to the low-pass domain, and to determine the filter prototype. Transformations of the low-pass prototype to the needed domain lead to the final network.

Since $H(0)<1$, Legendre filter cannot be realized as LC ladder network with equal terminations. If the passband ripple $\varepsilon$ and filter degree are known and $R_{G}=1$, then for $R_{L}$ we get:

$$
R_{L}=1+2 \delta^{2} \pm 2 \delta \sqrt{1+\delta^{2}}
$$

where

$$
\delta=\varepsilon \frac{\sqrt{2 L_{2 n}(0)}}{n+1} \frac{\left(\omega_{0}^{2}-1\right)^{m}}{\omega_{0}^{2 m}} .
$$

Proof Substituting $\omega=0$ into equation (2), we get

$$
\frac{P_{\max }}{P_{L}}=1+\frac{2 \varepsilon^{2} L_{2 n}(0)}{(n+1)^{2}} \frac{\left(\omega_{0}^{2}-1\right)^{2 m}}{\omega_{0}^{4 m}} .
$$

On the other hand

$$
\frac{P_{\max }}{P_{L}}=\frac{V_{G}^{2}}{4} \frac{R_{L}}{V_{0}^{2}}=\frac{\left(1+R_{L}\right)^{2}}{4 R_{L}} .
$$

After some algebraic manipulation it is obtained

$$
\frac{P_{\max }}{P_{L}}=1+\frac{\left(1-R_{L}\right)^{2}}{4 R_{L}} .
$$

From (16) and (18) to get one equation

$$
\frac{\left(1-R_{L}\right)^{2}}{4 R_{L}}=\left(\frac{\varepsilon \sqrt{2 L_{2 n}(0)}}{n+1} \frac{\left(\omega_{0}^{2}-1\right)^{m}}{\omega_{0}^{2 m}}\right)^{2}
$$

with unknown $R_{L}$. The result follows.

By equating (2) and (4) we get:

$$
|\Gamma(\omega)|^{2}=1-\frac{1}{1+\frac{2 \varepsilon^{2}\left(\omega_{0}^{2}-1\right)^{2}}{(n+1)^{2}} \frac{L_{2 n}\left(\omega^{2}\right)}{\left(\omega^{2}-\omega_{0}^{2}\right)^{2}}} .
$$

Reflection coefficient, $\Gamma(s)$, can be derived from $\left.\Gamma(s) \Gamma(-s)\right|_{\omega=-\mathrm{j} s}=|\Gamma(\omega)|^{2}$. Function $\Gamma(s)$ can be extracted from $\Gamma(s) \Gamma(-s)$ using following properties: $\Gamma(s)$ and $\Gamma(-s)$ have opposite poles and opposite zeros, and the poles of $\Gamma(s)$ lie in the left half of the $s$-plane, i.e. the denominator polynomial of $\Gamma(s)$ is a Hurwitz polynomial. For the minimum phase system, all zeros of $\Gamma(s)$ lie also in the left half of the $s$-plane.

Equation (3) may be rearranged to yield 


$$
Z_{\text {in }}(s)=R_{G} \frac{1-\Gamma(s)}{1+\Gamma(s)}=R_{G} \frac{D_{e}+D_{0}}{N_{e}+N_{0}} .
$$

Since $Z_{\text {in }}(s)$ is driving point of an LC network, then it can be written as

$$
Z_{\text {in }}=\frac{\frac{z_{11} z_{22}-z_{12}^{2}}{R_{L}}+z_{11}}{\frac{z_{22}}{R_{L}}+1}=z_{11}-\frac{z_{12}^{2}}{z_{22}+R_{L}}
$$

where $z_{11}, z_{12}$ and $z_{22}$ are the open-circuit impedance parameters of the lossless network.

Equation (21) can be written as

$$
Z_{\text {in }}(s)=\frac{\frac{D_{0}}{N_{0}}+\frac{D_{e}}{N_{0}}}{\frac{N_{e}}{N_{0}}+1}
$$

where $R_{G}=1$. The $z_{11}$ and $z_{22}$ parameters are identified as

$$
z_{11}=\frac{D_{e}}{N_{0}}, z_{22}=R_{L} \frac{N_{e}}{N_{0}} .
$$

These equations identify the impedance parameters $z_{11}$ and $z_{22}$ in terms of the even and odd parts of numerator and denominator of driving point impedance. The synthesis of the approximation function is reduced to realizing $z_{11}\left(\right.$ or $\left.z_{22}\right)$ in such a way that the zeros are also realized. This step entails using the zero shifting technique [15]. Finally, we realize the impedance $z_{11}$ as lossless two-port network terminated by the resistance $R_{L}$. Our realizations are limited to cases in which the lossless two-port is a ladder.

\subsection{An example}

The seventh order rational transfer function with single pair of zeros at $\pm \mathrm{j} 1.41442$, for minimum stopband attenuation of $50 \mathrm{~dB}$, has following form

$$
H(s)=\frac{0.0769937\left(s^{2}+2.00058\right)}{s^{7}+2.05536 s^{6}+3.602 s^{5}+3.91985 s^{4}+3.29749 s^{3}+1.93419 s^{2}+0.762676 s+0.155466}
$$

then the reflection coefficient is

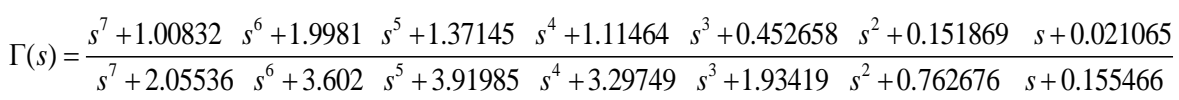

and the input impedance is obtained as $Z_{\text {in }}(s)=(1-\Gamma) /(1+\Gamma)$

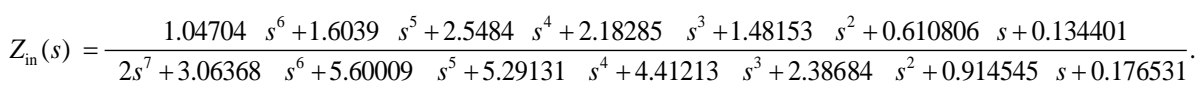


Factoring the numerator and denominator of $\Gamma(s)$ in even and odd parts and putting it in equation (24), we get

$$
\begin{aligned}
z_{11}(s)= & \frac{1.04704 s^{6}+2.5484 s^{4}+1.48153 s^{2}+0.134401}{2 s^{7}+5.60009 s^{5}+4.41213 s^{3}+0.914545 s} \\
& =\frac{0.523519\left(s^{2}+0.110914\right)\left(s^{2}+1.5994\right)\left(s^{2}+0.723594\right)}{s\left(s^{2}+0.327475\right)\left(s^{2}+0.872908\right)\left(s^{2}+1.59966\right)} .
\end{aligned}
$$

The impedance function $z_{11}$ must be realized to exhibit the zeros of transfer function at $\pm \mathrm{j} 1.41442$. The admittance function $y_{11}=1 / z_{11}$ has a pole at infinity. A part of the residue of this pole can be removed to create zero at $\pm \mathrm{j} 1.41442$. The necessary residue is shunt capacitor

$$
C_{1}=\left.\frac{y_{11}}{s}\right|_{s= \pm \mathrm{j} 1.41442}=1.49251
$$

The remainder admittance $y_{11}^{\prime}(s)$ has a pair of zeros at $s= \pm \mathrm{j} 1.8680664$

$$
y_{11}^{\prime}(s)=y_{11}(s)-s C_{1}=\frac{0.417642 s\left(s^{2}+0.511073\right)\left(s^{2}+1.59684\right)\left(s^{2}+2.00058\right)}{\left(s^{2}+0.110914\right)\left(s^{2}+0.723594\right)\left(s^{2}+1.5994\right)} .
$$

The impedance $z_{11}^{\prime}=1 / y_{11}^{\prime}$ has a pair of poles at $\pm \mathrm{j} 1.41442$, which can be removed by the partial fraction expansion

$$
k_{1}=\left.z_{11}^{\prime} \frac{s^{2}+2.00058}{s}\right|_{s= \pm \mathrm{j} 1.41442}=1.92669,
$$

and the remainder is

$$
z_{11}^{\prime \prime}=z_{11}^{\prime}-\frac{k_{1} s}{s^{2}+2.00058}=\frac{0.153631+0.830755 s^{2}+0.467711 s^{4}}{s\left(s^{2}+0.511073\right)\left(s^{2}+1.59684\right)} .
$$

In the above expression for $z_{11}^{\prime \prime}$, the term

$$
\frac{1.92669 s}{s^{2}+2.00058}=\frac{1}{0.519026 s+\frac{1}{0.963063 s}}
$$

can be realized as parallel resonant circuits as shown in Figure 4, which is realized by $C_{2}=0.519026$ and $L_{2}=0.963063$.

The remainder admittance of LC ladder network, $y_{11}^{\prime \prime}$, is

$$
y_{11}^{\prime \prime}=\frac{2.13807 s\left(s^{2}+0.511073\right)\left(s^{2}+1.59684\right)}{\left(s^{2}+0.209682\right)\left(s^{2}+1.56653\right)}
$$

and it can be realized by continued partial fraction expansion as 


$$
y_{11}^{\prime \prime}=2.13807 s+\frac{1}{1.41002 s+\frac{1}{2.3166 s+\frac{1}{1.08697 s+\frac{1}{0.857445}}}} .
$$

Thus, $C_{3}=2.13807, L_{4}=1.41002, C_{5}=2.3166, L_{6}=1.08697$ and $C_{7}=0.857445$. Finally, the equation (15) is used to determine $R_{L}=0.761343$. The final circuit is shown in Figure 4.

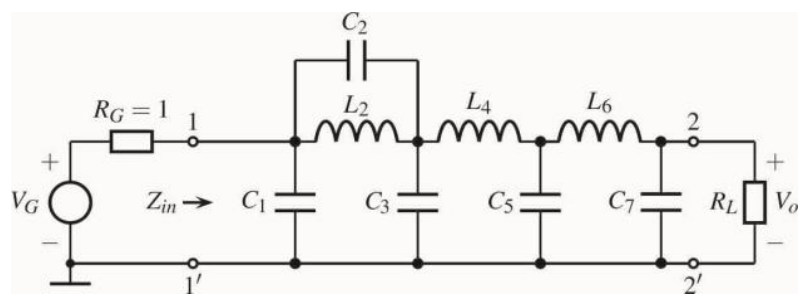

Fig. 4 Ladder circuit of the proposed new type filter for $n=7$ and $m=1$.

\section{COMPARISON WITH OTHER TyPes OF MONOTONIC PASSBAND MAGNITUDE RESPONSE}

A simple MATLAB computer program is used to find locations of the transmission zeros for any specified value of the minimum stopband attenuation. As an example, suppose that a minimum stopband attenuation of $50 \mathrm{~dB}$ is required. The seventh order new filter with one pair of imaginary-axis zeros satisfying this requirement has following poles:

$$
\begin{array}{ll}
s_{1}=-0.43542 \pm \mathrm{j} 0.47534, & s_{2}=-0.256318 \pm \mathrm{j} 0.821755 \\
s_{3}=-0.0802703 \pm \mathrm{j} 0.990445, & s_{4}=-0.511342
\end{array}
$$

and one pair of zeros at $z_{1,2}= \pm \mathrm{j} 1.41442$. Figure 5 shows corresponding pole-zero plot. Pole-zero plot of the inverse Chebyshev filter for $n=7$ and $50 \mathrm{~dB}$ stopband attenuation is also given for comparison.

The inverse Chebyshev filters should be prepared to be suitable for comparison with a present design. We first define $\omega_{c}$, as the cutoff frequency at which the squared magnitude is down to $1 /\left(1+\varepsilon^{2}\right)$. For convenience, we will set $\varepsilon$ to 1 , as has been used in the presented design. Then at $\omega_{c}$

$$
\left|F_{I I}\left(\mathrm{j} \omega_{c}\right)\right|^{2}=\frac{\delta^{2} T_{n}^{2}\left(1 / \omega_{c}\right)}{1+\delta^{2} T_{n}^{2}\left(1 / \omega_{c}\right)}=\frac{1}{1+\varepsilon^{2}},
$$

where $1 / \mathrm{A}^{2}=\delta^{2} /\left(1+\delta^{2}\right)$ is the maximum of the ripple amplitude in the stopband. Solving (37) for $\omega_{c}$ we have

$$
\omega_{c}=\frac{1}{\cosh \left(\frac{1}{n} \operatorname{arccosh} \sqrt{A^{2}-1}\right)} .
$$

Second, as a criterion for comparison we have used the cutoff slope: 


$$
\left.\frac{\mathrm{d}}{\mathrm{d} \omega}\left|F_{I I}(\omega)\right|^{2}\right|_{\omega=\omega_{c}}=-\frac{n}{2} \frac{\frac{1}{\omega_{c}^{2}}}{\sqrt{\frac{1}{\omega_{c}^{2}}-1}} \frac{\sqrt{\frac{1}{\delta^{2}}-1}}{\frac{1}{\delta}} .
$$

From equation (39) it can be concluded that the cutoff slope of inverse Chebyshev filters is close to the cutoff slope of Butterworth filters.

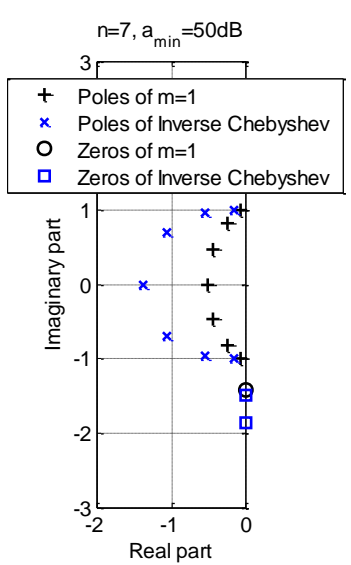

Fig. 5 Pole zero plot of the proposed and Inverse Chebyshev filter for $n=7$.
The magnitude and group delay responses for the proposed design, for the inverse Chebyshev filter and for the sum of squares Legendre polynomials all-pole filter, are shown in Fig. 6. It can be concluded that the magnitude response of the proposed design of passband monotonic filters is superior both in the passband and in the stopband. On the other side, the group delay characteristic of the inverse Chebyshev filter is slightly better.

Cutoff slopes for the proposed design, all-pole sum-of-square Legendre and inverse Chebyshev filter are: 64.281, 45 and 9.280, respectively. If $m=3$ the cutoff slope is slightly increased to 67.036 , but for $m=4$ it decreases to the value 64.625. Thus, best solution is for $m=2$. A disadvantage of the Inverse Chebyshev characteristic is that the passive realization requires more elements since have to be realized. A seventh degree passive ladder filter has six transmission zeros which have to be realized with seven LC parallel resonant circuits.

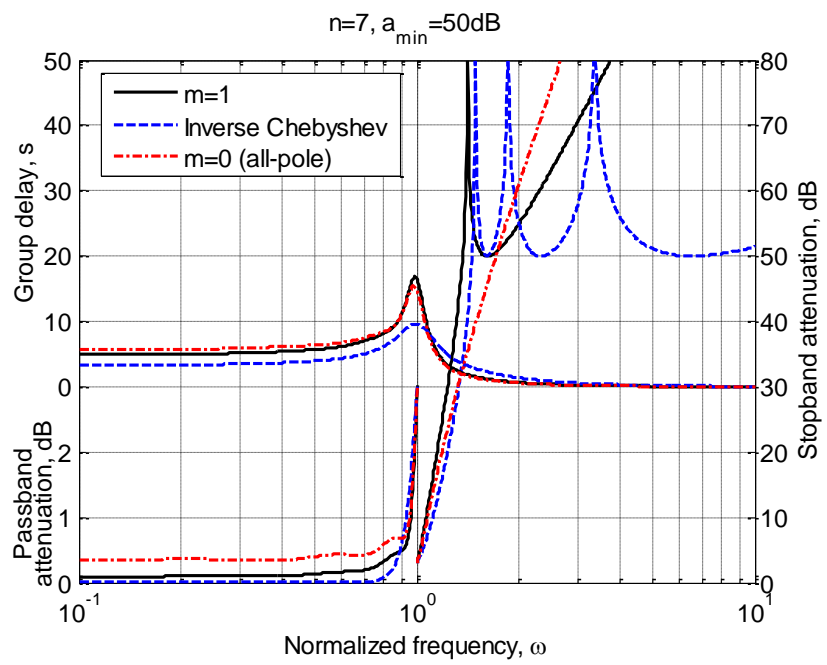

Fig. 6 Comparison of the proposed passband monotonic filter with inverse

Chebyshev filter and sum-of-squares Legendre (allpole) filter for $n=7$. 


\section{CONCLUSION}

New type of transfer function with monotonic response in the passband and nonmonotonic response in the stopband has been proposed. The stopband and passband performance of the filter can be improved by adding one simple or multiple pair of real frequency transmission zeros. For any prescribed minimum stopband attenuation the zero location can be determined.

In comparison with nearly monotonic allpole filters, new filters offer the following advantages: monotonic performances in the passband, smaller passband insertion loss, and greater cutoff slope.

Presented approximation is compared with all-pole transfer function and inverse Chebyshev filter on example for the seventh order lowpass filter. As revised by the results of comparison, the approximation with a double pair zeros on the imaginary axis yields, in many cases, the best solution in respect to the passband loss or to the cutoff characteristic.

A numerical example, which includes element values for an unequally terminated LC ladder network realization of these filters, is given to illustrate this method.

Acknowledgement: The work presented here was partially supported by the Ministry of Education, Science and Technological development of Republic of Serbia, under the project TR33008 - "A new approach to modeling of cable accessories in order to increase the efficiency of power transmission lines", and also under the project III 44004 -"Development and integration of design technology of intelligent mechatronic interface for medical applications (Humanism)".

\section{REFERENCES}

[1] T. L. Deliyannis, Y. Sun, and J. K. Fidler, Continuous-Time Active Filter Design. Boca Raton: CRC Press LLC, 1999.

[2] S. C. D. Roy, "Modified Chebyshev lowpass filters," International Journal of Circuit Theory and Applications, vol. 38, no. 5, pp. 543-549, Jun. 2009.

[3] H. G. Dimopoulos and E. Sarri, "The modified Pascal polynomial approximation and filter design method," Int. J. Circ. Theor. Appl., vol. 40, no. 2, pp. 145-163, Feb. 2012.

[4] S. Prasad, L. G. Stolarczyk, J. R. Jackson, and E. W. Kang, "Filter synthesis using Legendre polynomials," Proc. IEE, vol. 114, no. 8, pp. 1063-1064, Aug. 1967.

[5] A. Papoulis, "Optimum filters with monotonic response," Proceedings of the IRE, vol. 46, no. 3, pp. 906-609, Mar. 1958.

[6] W. Thomson, "Delay networks having maximally flat frequency characteristics," Proc. IEEE, vol. 96, no. part 3, pp. 487-490, 1949.

[7] D. Živaljević, N. Stamenković, and V. Stojanović, "Nearly monotonic passband low-pass filter design by using sum-of-squared Legendre polynomials," International Journal of 10 Circuit Theory and Applications, vol. 44, no. 1, pp. 147-161, 2016. [Online]. Available: http://dx.doi.org/10.1002/cta.2068

[8] M. Abramowitz and I. Stegun, Handbook of Mathematical Functions with Formulas, Graphs, and Mathematical Tables, 9th ed. New York, Dover: National Bureau of Standards Applied Mathematics Series 55, 1972.

[9] D. Johnson and J. Johnson, "Low-pass filters using ultraspherical polynomials," IEEE Trans. on Circuits Theory, vol. 13, no. 4, pp. 364-369, 1966.

[10] H. J. Orchard, "Inductorless filters," Electronics Letters, vol. 2, no. 6, pp. 224-225, Sep. 1966

[11] N. Balabanian, Network Synthesis. New York: Prentice-Hall, 1958.

[12] G. Daryanani, Principles of Network Synthesis and Design. New York: Jon Wiley and Sons, 1976.

[13] R. Raut and M. N. S. Swamy, Modern Analog Filter Analysis and Design - A Practical Approach. Weinheim, Germany: Wiley-VCH, 2010.

[14] A. Fettweis, "Digital filter structures related to classic filter networks," AEU, vol. Band 25, no. 2, pp. 79-89, Feb. 1971. 
[15] T. Rathore, “A zero-shifting technique and its applications,” IETE Technical Review, vol. 2, no. 7, pp. 231-237, 1985. [Online]. Available: http://dx.doi.org/10.1080/02564602.1985.11437792

[16] D. Živaljević, N. Stamenković, J. Živanić, "Sharp Cutoff Filters with Monotonic Passband Response", 18th International Symposium on Electrical Apparatus and Technologies SIELA 2014, 29-31 May, 2014, Bourgas, Bulgaria, Proceedings of digests, pp: 141-142. 